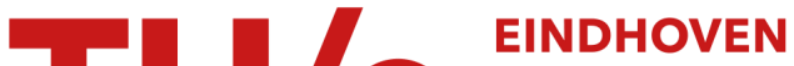 UNIVERSITY OF TECHNOLOGY
}

\section{Lift-up process in a heated-cylinder wake flow}

\section{Citation for published version (APA):}

Ren, M., Rindt, C. C. M., \& Steenhoven, van, A. A. (2006). Lift-up process in a heated-cylinder wake flow. Physics of Fluids, 18(1), 014106-1/12. https://doi.org/10.1063/1.2159031

DOI:

10.1063/1.2159031

Document status and date:

Published: 01/01/2006

\section{Document Version:}

Publisher's PDF, also known as Version of Record (includes final page, issue and volume numbers)

\section{Please check the document version of this publication:}

- A submitted manuscript is the version of the article upon submission and before peer-review. There can be important differences between the submitted version and the official published version of record. People interested in the research are advised to contact the author for the final version of the publication, or visit the $\mathrm{DOI}$ to the publisher's website.

- The final author version and the galley proof are versions of the publication after peer review.

- The final published version features the final layout of the paper including the volume, issue and page numbers.

Link to publication

\section{General rights}

Copyright and moral rights for the publications made accessible in the public portal are retained by the authors and/or other copyright owners and it is a condition of accessing publications that users recognise and abide by the legal requirements associated with these rights.

- Users may download and print one copy of any publication from the public portal for the purpose of private study or research.

- You may not further distribute the material or use it for any profit-making activity or commercial gain

- You may freely distribute the URL identifying the publication in the public portal.

If the publication is distributed under the terms of Article $25 \mathrm{fa}$ of the Dutch Copyright Act, indicated by the "Taverne" license above, please follow below link for the End User Agreement:

www.tue.nl/taverne

Take down policy

If you believe that this document breaches copyright please contact us at:

openaccess@tue.nl

providing details and we will investigate your claim. 


\title{
Lift-up process in a heated-cylinder wake flow
}

\author{
Maosheng Ren, ${ }^{\text {a) }}$ Camilo Rindt, and Anton van Steenhoven \\ Laboratory for Energy Technology, Department of Mechanical Engineering, Eindhoven University \\ of Technology, P.O. Box 513, 5600 MB Eindhoven, The Netherlands
}

(Received 1 June 2005; accepted 23 November 2005; published online 25 January 2006)

\begin{abstract}
A three-dimensional flow transition behind a heated cylinder is analyzed at low Reynolds numbers: $\operatorname{Re}=\mathcal{O}(100)$. Both visualizations and numerical simulations show that the transition manifests itself in the form of mushroom-type structures in the far wake and $\Lambda$-shaped structures in the near wake. The legs of the $\Lambda$-shaped structures coincide with streamwise vorticity regions. An intermediate stage is observed between the $\Lambda$-shaped structures and the escaping mushroom-type structures. This intermediate step is characterized by a lift-up process, which takes place in the center region between the legs and head of the $\Lambda$-shaped structures. As a result, hot fluid is being pulled out of the upper vortex core. Due to this lift-up process, mushroom-type structures are generated in the form of escaping vortex rings in the far wake. (C) 2006 American Institute of Physics.
\end{abstract}

[DOI: $10.1063 / 1.2159031]$

\section{INTRODUCTION}

Over the past 10 years, much attention has been paid to the three-dimensional (3D) vortex dynamics behind a cylinder. Experimental and theoretical studies of the 3D wake transition significantly contributed to the understanding of this dynamics. ${ }^{1,2}$ From a fundamental point of view, studies on the instability mechanisms resulting in the formation of two-dimensional (2D) and 3D vortical structures can contribute to a better understanding of the laminar-turbulent transition and may lead to ways to actively control the features of the flow.

For an unheated cylinder, Williamson's visualizations ${ }^{3}$ showed two modes of small-scale 3D instability. The two different modes scale on different features of the flow. For Reynolds numbers larger than 180-190, the mode-A instability has a spanwise wavelength of 4 cylinder diameters. Williamson suggested that the mode-A transition is associated with an elliptical instability of the primary vortex core during the process of shedding, which causes a deformation of the core. For Reynolds numbers larger than 230-260, a mode-B transition occurs with a spanwise wavelength of around 1 cylinder diameter. The mode- $\mathrm{B}$ instability is related with an instability of the shear layer in the braid region. In addition to the mode-A and mode-B transitions, Zhang et al. ${ }^{4}$ found a mode- $C$ transition with a spanwise wavelength of 1.8 cylinder diameters. Mode-C usually appears for $170<\operatorname{Re}<270$ only if mode-A and mode-B are inhibited to develop by a thin control wire placed parallel to the cylinder in the near wake.

Despite the fact that mixed convection around bluff bodies is of great importance for various engineering applications (electronics cooling, compact heat exchangers), wake stability for a heated cylinder has until now received very little attention compared to the forced convection case. For a horizontal mounted cylinder, the vortex shedding can be sup-

${ }^{a)}$ Electronic mail: m.ren@tue.nl pressed by heating of the cylinder at Reynolds values just above the critical value: $\operatorname{Re}_{c}=49$ (Lecordier et al., ${ }^{5}$ Wang et $\left.a l .{ }^{6}\right)$. In Lecordier et al., ${ }^{7}$ the sudden disappearance of the vortex shedding phenomenon is analyzed and it is concluded that the influence of the temperature on the viscosity is the primary source. There has been almost no understanding of the effect of a heat input on the behavior of the coherent structures behind a cylinder at low Reynolds numbers until the recent studies by Kieft et al. ${ }^{8,9}$ For Reynolds numbers around 80 , Kieft et al. ${ }^{10}$ found that, by heating the cylinder, the vortices in the upper and lower rows acquire different strengths due to baroclinic vorticity production. This strength difference increases for increasing Richardson numbers. It is observed that, for small Richardson numbers, the shed vortices move slightly downwards.

From an earlier study in our laboratory, ${ }^{11}$ it was shown that, when the cylinder is heated, a 3D transition in the wake flow occurs at a Reynolds number lower than for the unheated case. Escaping mushroom-type structures occur in the far wake with a spanwise distance of around twice the cylinder diameter. ${ }^{12}$ Furthermore, it has also been shown in Ref. 12 that the vortex formation shows quite large differences between the "in-plume" position (where the plume escapes further downstream) and the "out-of-plume" position (where the plume does not escape further downstream). The shedding process for the out-of-plume position is quite similar to the one for an unheated cylinder. For the in-plume positions, the formation of the saddle point in the upper and lower side of the wake is not symmetrical in one vortex shedding cycle. Furthermore, at the in-plume positions, an upward motion occurs directly behind the cylinder, which separates the vortices from the cylinder wall. It is observed that an upward motion near the cylinder wall enhances the formation of the upper vortices and weakens the formation of the lower ones.

For the forced convection case, the flow becomes threedimensional when the Reynolds number becomes larger than $\operatorname{Re}=180$. To distinguish this transition from the other transition modes, the 3D transition occurring for mixed convection 


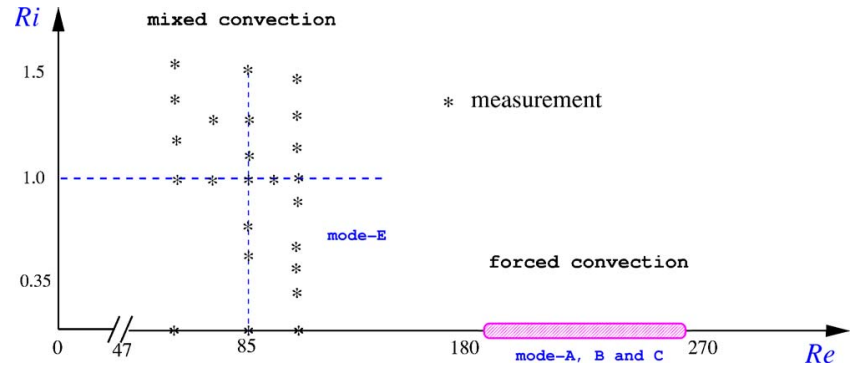

FIG. 1. (Color online). The 3D transition diagram as a function of Reynolds and Richardson numbers. The measurements are performed at discrete Reynolds and Richardson numbers, which are indicated with asterisks $(*)$.

is denoted as "mode-E" (see Fig. 1). The mode-E transition is located at a much lower Reynolds number. Table I shows the length scales of the different modes of 3D transition. It seems, from the jump in length scale, that there exist different kinds of 3D transition mechanisms. The goal of the present paper is to understand the mode-E transition mechanism.

It has been observed that the flow features of mode-E transition are similar within the range of Reynolds numbers $75<\operatorname{Re}<117$ and the range of Richardson numbers 0.35 $<\mathrm{Ri}<2.5$, although the measurements are performed at discrete Reynolds and Richardson numbers. The current investigation focuses on the flow phenomena at one Reynolds number and one Richardson number, $\mathrm{Re}=85$ and $\mathrm{Ri}=1.0$, respectively. Both experimental and numerical techniques are used, including an electrochemical tin-precipitation visualization method and a 3D spectral element method (3DSEM). The paper is organized as follows. First, a description of the problem definition is given, together with the experimental setup and investigation techniques. Then, global observations of the 3D transition behind a heated cylinder are presented. Next, the formation process of the mushroom-type structure is investigated. Finally, the main conclusions are given.

\section{METHODOLOGY}

\section{A. Problem definition}

In the current investigation the $3 \mathrm{D}$ transition behind a heated cylinder is investigated at low Reynolds numbers. The cylinder is exposed to a horizontal uniform cross flow (Fig. 2), where the $z$ axis is in spanwise direction, the $x$ axis is in streamwise direction, and the $y$ axis is in negative gravity direction. The cylinder is positioned at $x=0$ and $y=0$.

In the calculations, the flow is assumed to obey the Boussinesq approximation; the density variations are negli-

TABLE I. The different modes of 3D transition in the cylinder wake flow. Mode-E is investigated in the present study.

\begin{tabular}{lccc}
\hline \hline 3D transition & Reynolds number & Richardson number & Wavelength \\
\hline Mode-A (Ref. 3) & $180-230$ & 0 & $4 d$ \\
Mode-B (Ref. 3) & $200-280$ & 0 & $1 d$ \\
Mode-E & $75-117$ & $0.35-2.5$ & $2 d$ \\
\hline
\end{tabular}

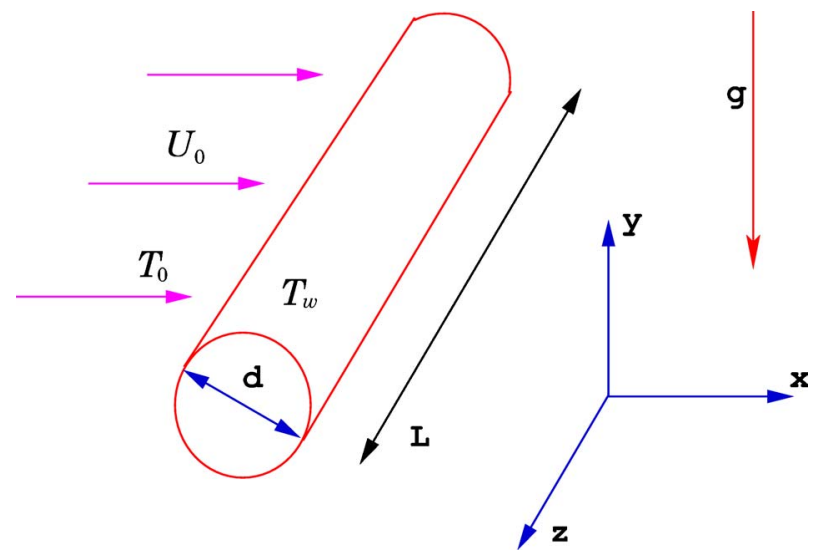

FIG. 2. (Color online). Problem definition: the cylinder, positioned at $x=0$ and $y=0$, has a temperature $T_{w}$ and is subject to a horizontal cross flow with velocity $U_{0}$ and temperature $T_{0}$ and gravity is in negative $y$ direction.

gible except in the buoyancy force. The dimensionless conservation equations for mass, momentum, and energy are, respectively,

$$
\begin{aligned}
& \nabla \cdot \mathbf{u}=0 \\
& \frac{\partial \mathbf{u}}{\partial t}+\mathbf{u} \cdot \nabla \mathbf{u}=-\nabla p+\frac{1}{\operatorname{Re}} \nabla^{2} \mathbf{u}-\operatorname{Ri} \Theta \mathbf{g}, \\
& \frac{\partial \Theta}{\partial t}+\mathbf{u} \cdot \nabla \Theta=\frac{1}{\operatorname{Re} \operatorname{Pr}} \nabla^{2} \Theta
\end{aligned}
$$

with $\mathrm{Re}=U_{0} d / \nu$ the Reynolds number, $\mathrm{Ri}=\mathrm{Gr} / \mathrm{Re}^{2}=g \beta\left(T_{w}\right.$ $\left.-T_{0}\right) d / U_{0}^{2}$ the Richardson number, $\mathrm{Gr}=g \beta\left(T_{w}-T_{0}\right) d^{3} / \nu^{2}$ the Grashof number, $\operatorname{Pr}=\nu / \kappa$ the Prandtl number, $\beta$ the thermal expansion coefficient, $\kappa$ the thermal diffusivity, $g$ the gravity constant, $\mathbf{g}=(0,-1,0)^{T}$ the dimensionless gravity vector, $\Theta$ $=\left(T-T_{0}\right) /\left(T_{w}-T_{0}\right)$ the dimensionless temperature, $\mathbf{u}$ the dimensionless velocity, and $p$ the dimensionless pressure. From these dimensionless conservation equations with suitable boundary conditions, one can conclude that the character of the flow depends on the dimensionless parameters Re, $\mathrm{Ri}$, and Pr. In the current investigation, $\mathrm{Ri}$ is equal to 1.0 and $\mathrm{Re}$ is set to 85 . Because water is used as the working fluid, the Prandtl number is around 7.

\section{B. Experimental setup and visualization method}

The apparatus used in the experiment consists of three parts: the water tank, the light source with illumination system, and the image recording with post-processing system.

The flow is investigated in a so-called towing tank configuration, as shown in Fig. 3, with dimensions length $\times$ width $\times$ height $=500 \times 50 \times 75 \mathrm{~cm}^{3}$. In this configuration the cylinder is towed through the water with a constant velocity $U_{0}$. The cylinder has a length of $L=495 \mathrm{~mm}$ and a diameter of $d=8.5 \mathrm{~mm}$ (aspect ratio $L / d=58$ ). The test section consists of $15 \mathrm{~mm}$ thick glass windows, held together by a steel frame, so the towing tank is optically accessible from all directions. The cylinder is positioned between two perspex plates, which are connected to a stiff structure, which also carries the measurement equipment, such as cameras 


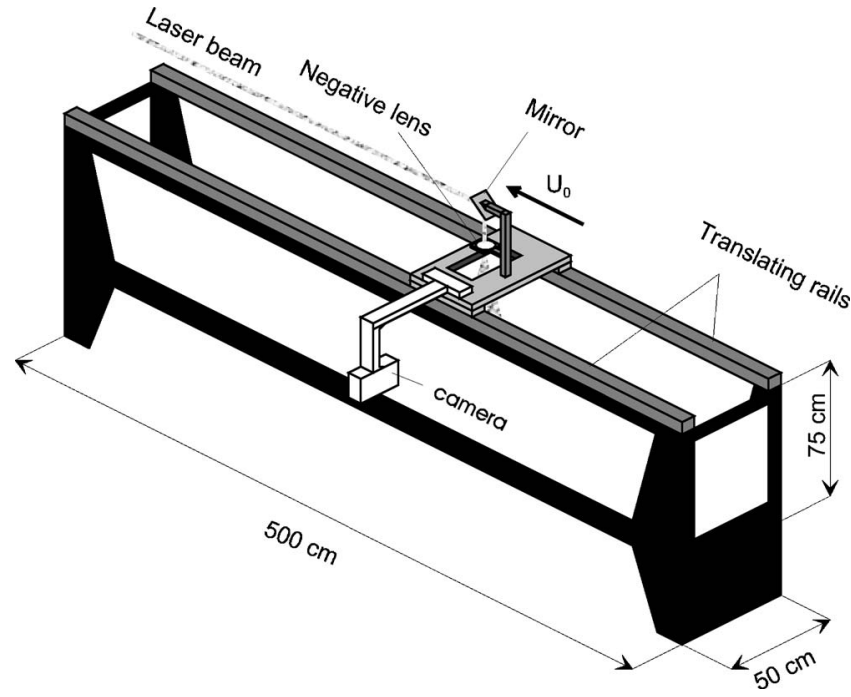

FIG. 3. Experimental setup. In this configuration the cylinder is towed through the water with a constant velocity $U_{0}$.

and light sources. The stiff construction can be translated along two rails that are mounted on top of the water tank. The perspex plates are constructed in such a way that minimum disturbances are created and oblique vortex shedding is suppressed. A cylindrical heating element with a diameter of $6.35 \mathrm{~mm}$ is used to obtain the desired cylinder wall temperature. The whole of the tank is supported by a sturdy steel framework and is situated in a temperature-controlled laboratory. A detailed description of the towing tank is given in Refs. 13 and 14.

For the light source, a pulsed neodymium:yttriumaluminum-garnet (Nd:YAG) laser is used. The laser emits light with a wavelength of $532 \mathrm{~nm}$. One laser pulse has a duration of $6 \mathrm{~ns}$ and a maximum energy of $200 \mathrm{~mJ}$. The laser beam is directed parallel to the bottom of the water tank, as shown in Fig. 3. The beam passes a negative lens to form a thin laser sheet. The laser is triggered by a camera and operates at $29 \mathrm{~Hz}$.

The recording is performed by a CCD camera (Kodak Megaplus, 10-bit ES 1.0, $1008 \times 1019$ pixels $^{2}$ ). The camera records images at a maximum frame rate of $29 \mathrm{~Hz}$. A Nikon lens with a focal distance of $55 \mathrm{~cm}$ is used in front of the CCD camera. A detailed description of the image recording and post-processing system is given in Ref. 15 .

Flow visualizations are carried out using an electrochemical tin-precipitation method (Honji et al. ${ }^{16}$ ). In this method tin ions are separated from an anode by applying a voltage difference. A thin tin wire that is used as an anode in the current setup is positioned upstream of the cylinder. Because the tin ions do not dissolve in $p \mathrm{H}$-neutral water, the small tin-hydroxide particles of $\mathcal{O}(1 \mu \mathrm{m})$ form a homogeneous sheet that moves towards the cylinder. With this sheet the wake behind the cylinder is visualized. A detailed description of the visualization method is presented in Ref. 11.

\section{3D spectral element method}

Besides the visualization experiments, 3D numerical simulations were performed. For an efficient temporal dis-

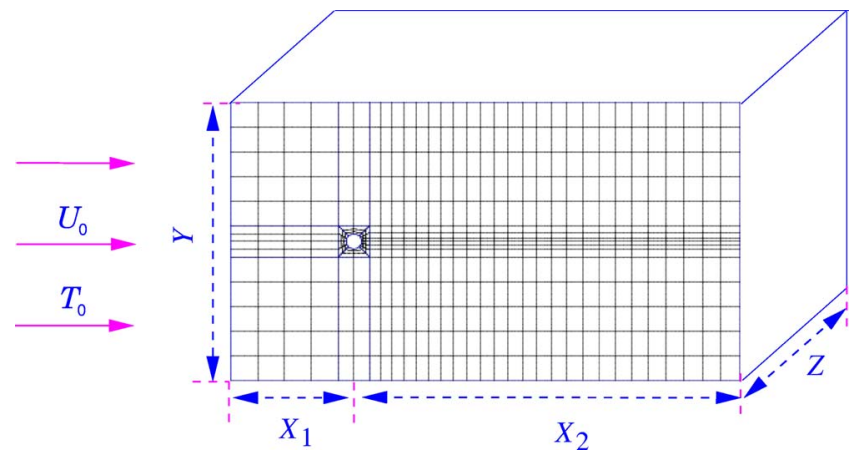

FIG. 4. (Color online). Spectral element grid with dimensions length $\times$ height $\times$ width $=33 d \times 18 d \times 4 d$, consisting of 1880 elements and 252315 node points (fifth-order approximation polynomial per element).

cretization of Eqs. (1)-(3), a splitting operation on the convection-diffusion equations is applied (Maday et al. ${ }^{17}$ ). As a result, the convection and the diffusion operators are treated separately. For the momentum equation, in combination with the continuity equation, a pressure correction method is applied. This results then in three steps, which account for the convection, diffusion, and pressure terms, respectively. The convection equation is integrated forward in time by an explicit third-order Taylor-Galerkin scheme, which comprises of three explicit time steps within one implicit time step. The diffusion equation is discretized by an implicit second-order backwards difference scheme. The pressure term is treated by a projection method. ${ }^{18}$ Due to the limitation of memory storage, an iterative technique is used and the linear system is solved based on a preconditioning conjugate gradient method. The energy equation is solved in a similar way.

For the spatial discretization, a high-order SEM is used (Karniadakis and Triantafyllou ${ }^{19}$ ). The SEM can be considered as a mixture of a finite element method and a spectral method. The principle of SEM is rather straightforward. Within an element, the equations are discretized using a high-order spectral method. Combining the solutions obtained in all these elements results in the solution on the whole domain. Furthermore, special consideration has been given to the continuity of the solution over the element boundaries. The decomposition of the domain into elements gives the method the geometric flexibility. By using highorder approximation functions within one element, a spectral convergence rate and small numerical errors, such as numerical dispersion and diffusion, can be achieved. More information on the validation of the numerical scheme can be found in Ref. 18.

The element distribution used in the calculation is shown in Fig. 4. The boundary conditions are prescribed as follows. At the inlet and cylinder wall Dirichlet boundary conditions are applied for both velocity components and the temperature. The normal velocity and the tangential stresses at the side, top, and bottom walls are set to zero. Also the temperature is set to zero here. At the domain outlet, homogeneous Neumann conditions are prescribed for the normal and tangential velocity components as well as for the temperature. The boundary conditions for the pressure correction follow 


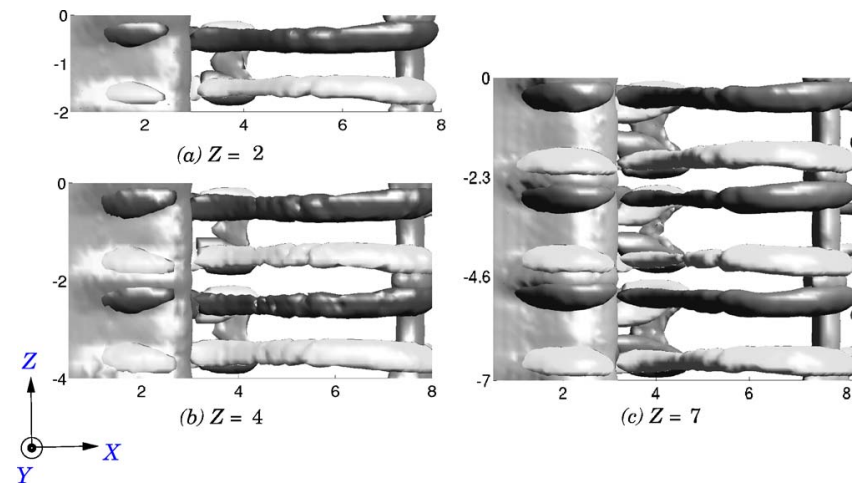

FIG. 5. (Color online). Calculated coherent structures for $\mathrm{Re}=85$ and $\mathrm{Ri}$ $=1.0$, for domain sizes $Z=2, Z=4$, and $Z=7$. (a)-(c) Top view of isosurface $\omega_{x}= \pm 0.4$ (streamwise vorticity) and isotemperature surface $\Theta=0.15$.

immediately from the global mass conservation. This implicitly states that the pressure is near zero at the outflow boundary. ${ }^{18}$ It should be noticed that the vortices leaving the computational domain will be influenced by the applied boundary condition at the outflow boundary. However, van de Vosse $e t a l .{ }^{20}$ showed that this influence is hardly noticeable.

The validation is carried out by studying the effect of the domain size and the grid resolution for an unheated flow at $\mathrm{Re}=100.2 \mathrm{D}$ flow simulations should adequately reproduce the results of experimental measurements. In literature an often-quoted test for the accuracy for cylinder flow computations is the Strouhal number as function of the Reynolds number. The validation studies show that the Strouhal number increases with decreasing inflow length $X_{1}$, decreases with decreasing outflow length $X_{2}$, and increases with decreasing domain height $Y$. Similar tendencies have been reported in Ref. 19.

In $3 \mathrm{D}$ calculations, the solutions are affected by the used span length $Z$ of the computational domain. Therefore the influence of the spanwise length on the coherent structures is studied at $\mathrm{Re}=85$ and $\mathrm{Ri}=1.0$. At this Reynolds and Richardson number, the previous experimental results indicate that there exists an averaged spanwise wavelength $2 d$ ( $d$ being the cylinder diameter). The validation studies show that the Strouhal number slightly increases with an increasing span length $Z .{ }^{14}$ The spanwise wavelength as found from the calculations is more or less fixed by the type of the boundary conditions prescribed at the side walls.

Figure 5 presents secondary coherent structures in order to distinguish the dependence of the calculated spanwise wavelength on the span length of the computational domain. It appears that, for a spanwise domain size of $Z=2$ and $Z$ $=4$, a spanwise wavelength of exactly $2 d$ is found, which can be deduced from the streamwise vorticity isosurface $\omega_{x}$ $= \pm 0.4$ [see Figs. 5(a) and 5(b)]. This value is in agreement with the experimental observations of the averaged distance between the mushroom-type structures. However, for a larger domain size $Z=7$, a spanwise wavelength of $7 / 3 \simeq 2.3 d$ is found [see Fig. 5(c)]. Nevertheless, the global flow structures show similar features, such as the escape of the mushroomtype structures from the upper vortices in the far wake. In

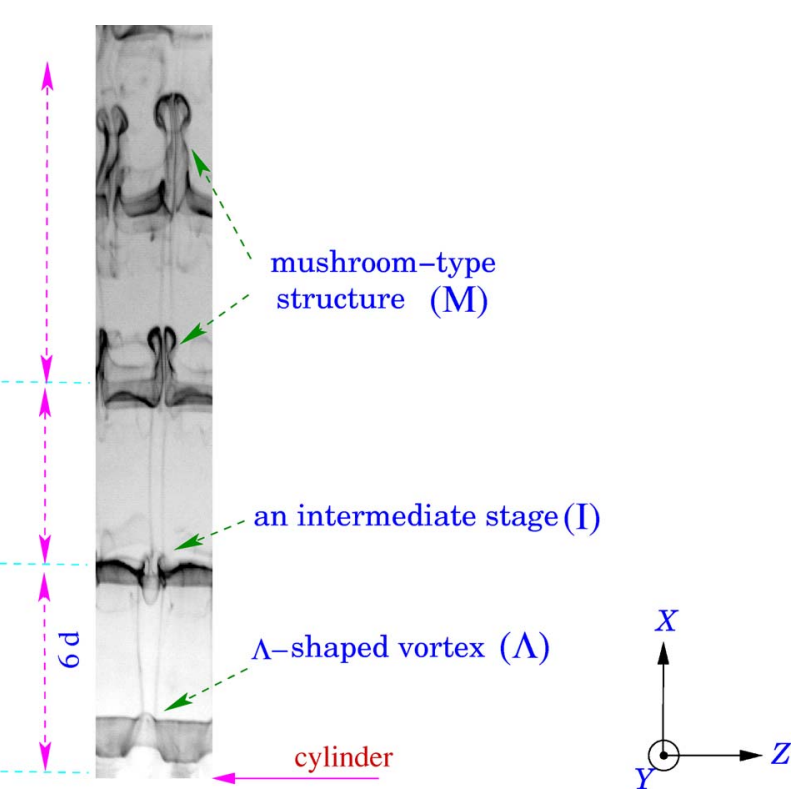

FIG. 6. (Color online). Top view of the wake flow pattern obtained by tin-precipitation visualization at $\mathrm{Re}=117$ and $\mathrm{Ri}=1.0$. The flow is from bottom to top and the gravity direction is in the negative $y$ direction.

this paper the results are shown for a computational domain with dimensions length $\times$ height $\times$ width $=33 d \times 18 d \times 4 d$.

\section{COHERENT STRUCTURES IN THE FLOW FIELDS}

For $\mathrm{Re}=117$ and $\mathrm{Ri}=1.0$, the $3 \mathrm{D}$ transition manifests itself in the form of escaping mushroom-type structures in the far wake. ${ }^{12}$ Figure 6 shows a top view of the wake flow pattern, obtained by means of the tin-precipitation visualization technique. The flow is from bottom to top and the gravity direction is in the negative $y$ direction.

It is observed that a mushroom-type structure, which is marked as $\mathbf{M}$, escapes from the upper vortex and stretches in negative gravity direction. In the far wake the mushroomtype structure develops into the shape of a vortex ring. Furthermore, a $\Lambda$-shaped structure is observed closely behind the cylinder, marked as $\Lambda$ (see Fig. 6).

The $\Lambda$-shaped structure has the same spanwise position as the mushroom-type structure. Furthermore, connection lines can be observed between the $\Lambda$-shaped structure and the mushroom-type structure. This suggests that the formation of the mushroom-type structure is strongly linked with the $\Lambda$-shaped structure in the near wake. Moreover, an intermediate stage marked as I (see Fig. 6) is located between the development of the $\Lambda$-shaped structure and the mushroomtype structure.

Additionally, a numerical simulation has provided a detailed insight into the coherent structure formation and evolution process towards the far wake. Figure 7 shows the calculated flow structures by using the iso- $\lambda_{2}$ surface $\lambda_{2}$ $=-0.05$ for $\mathrm{Re}=85$ and $\mathrm{Ri}=1.0$. Here the $\lambda_{2}$ definition is used to identify the flow topology and vortical structures. ${ }^{14,21}$ 
(a)

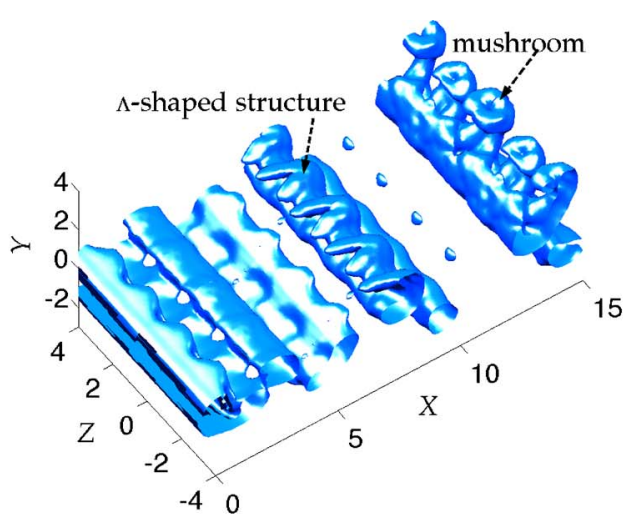

(b)

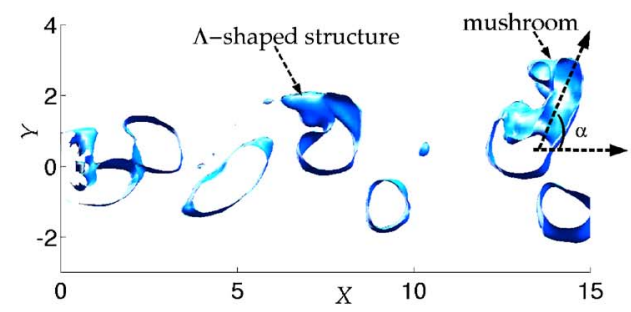

(c)

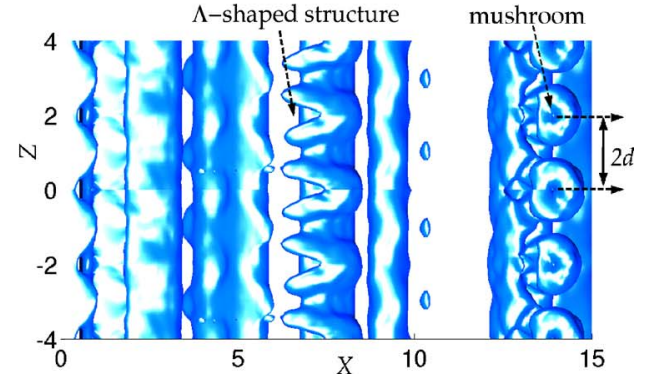

FIG. 7. (Color online). Numerically calculated iso- $\lambda_{2}$ surfaces $\lambda_{2}=-0.05$, which is about $2 \%$ of its minimum value, at $t=2 T / 4$ for $\mathrm{Ri}=1.0$ and $\mathrm{Re}$ =85. (a) Perspective view; (b) side view; (c) top view (enhanced online).

It has to be noted that the experiments and the calculation are performed at different Reynolds numbers. However, similar coherent structures have been found from both approaches.

In Fig. 7(a), mushroom-type structures are observed in the far wake. The mushroom-type structures are located above the upper vortices. The core area of each mushroomtype structure has an inclination angle of approximately $\alpha$ $\simeq 65^{\circ}$, as shown in Fig. 7(b). Figure 7(c) indicates that the spanwise distance of the mushroom-type structures is $2 d$.

In the near wake, $\Lambda$-shaped structures are observed at $x=7.5$ [see Fig. 7(c)]. The $\Lambda$-shaped structures consist of two legs and a head. Furthermore, it is observed that the $\Lambda$-shaped structures are located above the cores of the upper vortices [see Fig. 7(b)].

From both the visualization and numerical results, it can be concluded that the spanwise positions of the $\Lambda$-shaped structures are the same as the positions of the mushroomtype structures. The route map of the transition process can be distinguished into two steps: the $\Lambda$-shaped structure and the mushroom-type structure (see Fig. 8). It seems that the legs of the $\Lambda$-shaped structure coincide the regions of streamwise vorticity, which will be discussed in Sec. IV. Next, the attention will be mainly focus on the transition process from a $\Lambda$-shaped structure towards a mushroom-type

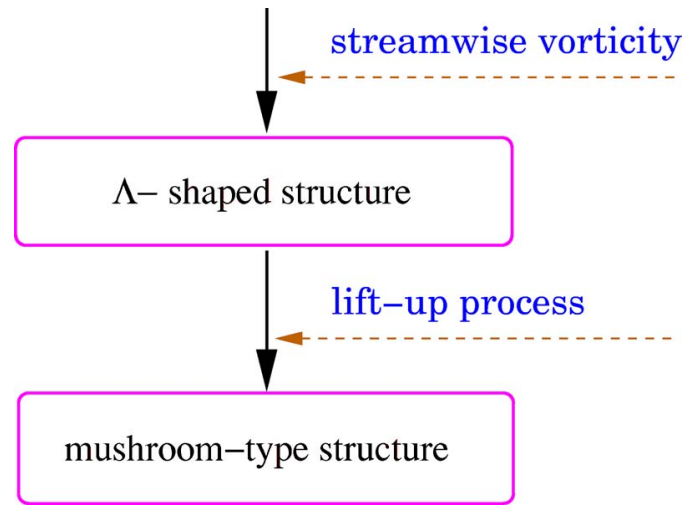

FIG. 8. (Color online). The route map of the transition process.

structure. This intermediate step is characterized by a lift-up process (Sec. V), which takes place at the regions between the legs and head of the $\Lambda$-shaped structure.

\section{THE FORMATION OF THE $\Lambda$-SHAPED STRUCTURE}

\section{A. A global view}

Figure 9 shows a top view visualization of the wake flow pattern at $\mathrm{Re}=117$ and $\mathrm{Ri}=1.0$. In Fig. 9(a), the forming upper vortex is observed at about two cylinder diameters downstream of the rear end of the cylinder. It is observed that the upper vortex is more or less parallel to the cylinder axis. In Fig. 9(b), the development of a concave shape is observed. It is seen that this concave shape further develops into a $\Lambda$-shaped structure [see Fig. $9(\mathrm{~d})]$.

Furthermore, there are two long filaments behind the head of the $\Lambda$-shaped structure. These thin lines connect the primary vortices and are aligned in streamwise direction. It seems that these connection lines are related to the streamwise vorticity in the braid region, as will be shown in Fig. 11. Besides, Figs. 9(i) and 9(j) show a small amplitude spanwise oscillation along these filaments.

Figure 10 shows the development of the numerically calculated coherent structures, captured by the iso- $\lambda_{2}$ surface $\lambda_{2}=-0.05$, as function of time at $\mathrm{Re}=85$ and $\mathrm{Ri}=1.0$. In Fig. $10(\mathrm{e})$, the $\Lambda$-shaped structure is clearly observed at position $x=6.0$ and it has two legs and a head.

For $t=3 T / 6$, the $\Lambda$-shaped structure is found at about $x$ $=4.5$ and for $t=2 T / 6$ at $x=3.75$. For $t=T / 6$ the $\Lambda$-shaped structure is hardly visible, although a distinct "concave shape" [Fig. 10(b)] appears around the rear end of the upper vortex at $x=2.5$. For $t=0$, the rear end of the upper vortex has a "convex shape" [Fig. 10(a)] at $x=2.0$.

Between time $t=0$ and $t=T / 6$, the shape of the rear end of the upper vortex changes from convex towards concave. This suggests that the formation of the $\Lambda$-shaped structure occurs somewhere in this time interval. A similar concave shape is also found in the visualization results, as shown in Fig. 9(b).

Furthermore, Fig. 10(a) shows a so-called "valley region" close behind the cylinder. From Ref. 14 it is known that a valley region is characterized as an area of low-speed fluid with a high temperature. Possibly, the occurrence of the 


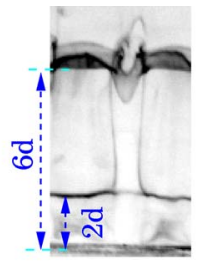

(a)

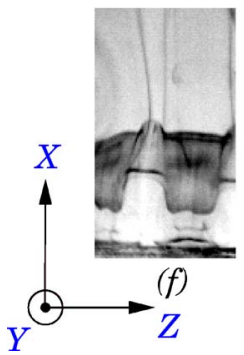

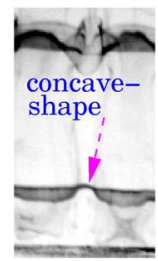

(b)

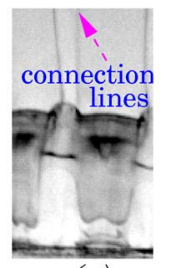

(g)

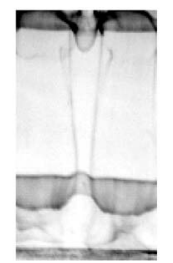

(c)

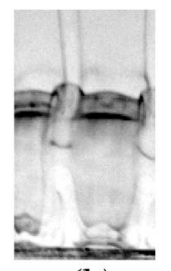

(h)

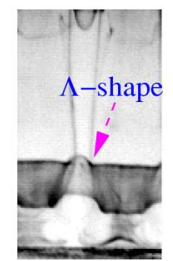

(d)

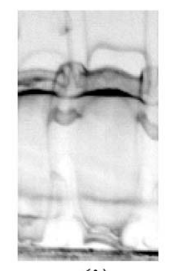

(i)

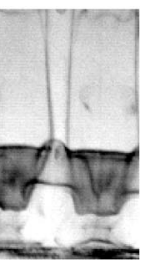

(e)

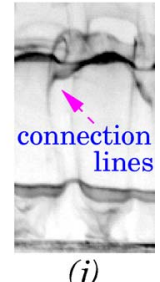

FIG. 9. (Color online). Top view of the wake flow pattern; the time step between pictures is $\Delta t=T / 10$, obtained by tin-precipitation visualization at $\mathrm{Re}=117$ and $\mathrm{Ri}=1.0$. The flow is from bottom to top and the gravity direction is in the negative $y$ direction. concave shape at the rear end of the upper vortex is associated with the fluid convected from the valley region into the upper wake.

\section{B. The $\Lambda$-shaped structure}

To understand the formation of the $\Lambda$-shaped structure, one has to determine the origin of the legs of the $\Lambda$-shaped structure. In Fig. 10, it is observed that the legs of the $\Lambda$-shaped structure are aligned in the streamwise direction. It appears that the legs of the $\Lambda$-shaped structure coincide with regions of relatively high streamwise vorticity. Therefore the evolution of the streamwise vorticity is studied, visualized with iso- $\omega_{x}$ surface $\omega_{x}= \pm 0.4$ in Fig. 11 , to gain insight into the formation process of the $\Lambda$-shaped structure.

It can be seen in Fig. 11(e) that the tip of the regions with streamwise vorticity is observed at $x \simeq 6.0$. These regions spatially coincide with the legs of the $\Lambda$-shaped structure, as observed in Fig. 10(e). Streamwise vorticity is generated in the vicinity of the cylinder. Together with the vortex shedding process, the regions with streamwise vorticity are advected downstream with the primary upper vortex, as shown in Fig. 11(a). Furthermore, the streamwise vorticity regions grow in size until the vorticity supply is cut off from the cylinder wall, which is indicated in Fig. 11(f).

Additionally, temperature isosurface $\Theta=0.15$ is added to visualize the position of the primary upper vortex. Figure 11(e) shows a "bulge shape" at $x=6.0$ and $z=-2.0$ in the upper vortex. This bulge shape suggests an accumulation of hot fluid. The bulge shape appears between the regions of relatively high streamwise vorticity.

However, at the lower half of the wake, the temperature distribution shows a different picture. In Fig. 11(f), a "void region" is observed at $x=3.0$ and $z=-2.0$. This void region suggests accumulation of cold fluid at the in-plume positions in the lower vortex core. It appears that the occurrence of the bulge and void region in the temperature distribution demonstrates the difference in effect of the streamwise vorticity regions in the upper and lower halves of the wake. In fact, the streamwise vorticity regions are positioned in the braid regions connecting the upper and lower vortices. At the up- per half of the wake, the braid region is located above the core of the upper vortex. Hot fluid from the vortex core is lifted up between the streamwise vorticity legs and accumulating around the bulge region. However, at the lower half of the wake, the braid region is located below the lower vortex. The streamwise vorticity legs bring cold fluid from outside the wake into the lower vortex core at the in-plume position.

To conclude, the above findings suggest that the streamwise vorticity regions located in the braid regions have a strong influence on the temperature distributions in the vortex cores.

\section{THE DEVELOPMENT TOWARDS MUSHROOM-TYPE STRUCTURES}

\section{A. A global view}

Figure 12 shows an oblique top-view visualization of the wake flow pattern at $\mathrm{Re}=117$ and $\mathrm{Ri}=1.0$. In the near wake a $\Lambda$-shaped structure, marked as $\Lambda$ in Fig. 12(a), is observed. Furthermore, a mushroom-type structure, marked as $\mathbf{M}$, is found in the far wake and an intermediate stage, marked as $\mathbf{I}$, is located between the $\Lambda$ and $\mathbf{M}$ structures. Additionally, there are two filaments connecting the $\Lambda$ and $\mathbf{M}$ structures.

To understand what happens in this intermediate stage, the coherent structures are visualized using the iso- $\lambda_{2}$ surface $\lambda_{2}=-0.05$. In Fig. 13, a top view of the flow pattern is shown for $\mathrm{Re}=85$ and $\mathrm{Ri}=1.0$.

Initially, the $\Lambda$-shaped structure $(\Lambda)$ is observed around $x=5.0$ in Fig. $13(\mathrm{~g})$, above the upper vortex. It is seen that gradually the legs of the $\Lambda$-shaped structure disappear. In Fig. $13(d)$, the head region of the $\Lambda$-shaped structure becomes a "half-torus" shape (H-T). Furthermore, this H-T suddenly develops into a mushroom-type structure (M) [see Figs. 13(b) and 13(c)] at $x=15.0$.

It might be clear from the above that all the different structures introduced, such as $\Lambda, \mathrm{H}-\mathrm{T}$, and $\mathrm{M}$, in fact all refer to different stages of the same flow structure evolution. The $\Lambda$-shaped structure is an early stage of the H-T structure and ultimately of the mushroom-type structure, forming the 

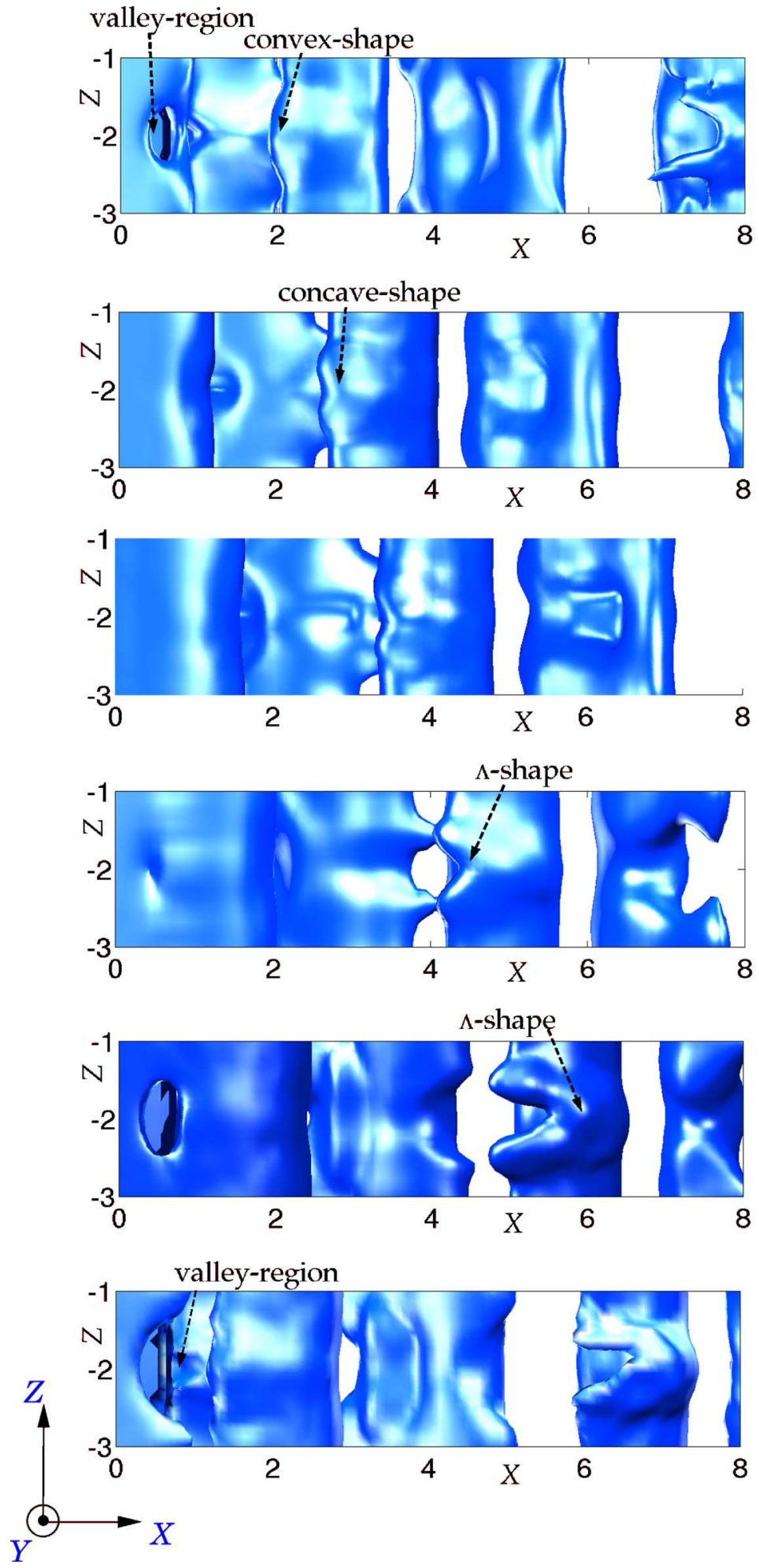

(a) $t / T=0$

(b) $t / T=1 / 6$

(c) $t / T=2 / 6$

FIG. 10. (Color online). Calculated iso- $\lambda_{2}$ surface $\lambda_{2}=-0.05$ as function of time at $\mathrm{Re}=85$ and $\mathrm{Ri}=1.0$. The flow is from left to right and the gravity direction is in the negative $y$ direction.

(d) $t / T=3 / 6$

(e) $t / T=4 / 6$

(f) $t / T=5 / 6$ vortex ring. It is reasonable to assume that the flow structure transformation from $\Lambda$ to H-T is related to a "lift-up" process. This lift-up process is also often encountered when discussing the evolution of hairpin structures in a boundary layer flow. ${ }^{22}$ To illustrate what happens in detail for the heated wake flow under consideration, the corresponding temperature isosurfaces are examined in Fig. 14 during this transformation process.

At the beginning, the temperature distribution has a bulge shape, indicated as B in Fig. 14(a), which is located at $x=7.5$ and $z=-2$ above the upper vortex. Furthermore, it can be seen that the bulge shape coincides with the region between the legs and head of the $\Lambda$-shaped structure. This suggests that, in the same way as the sketch in Fig. 16, the formation of the bulge shape is a result of hot fluid lifted up from the vortex core.

Furthermore, it is observed that the B region is continuously growing and becomes elongated in the $y$ direction. 


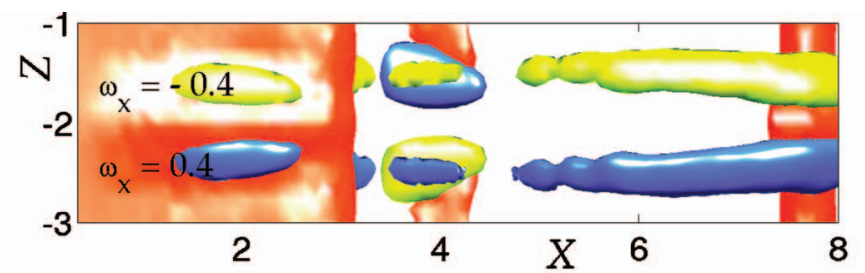

(a) $t / T=0$

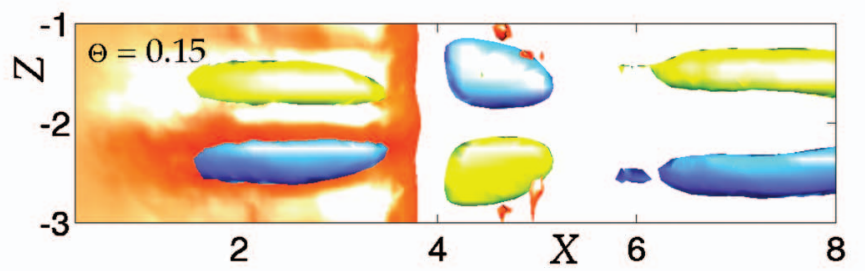

(b) $t / T=1 / 6$

(c) $t / T=2 / 6$

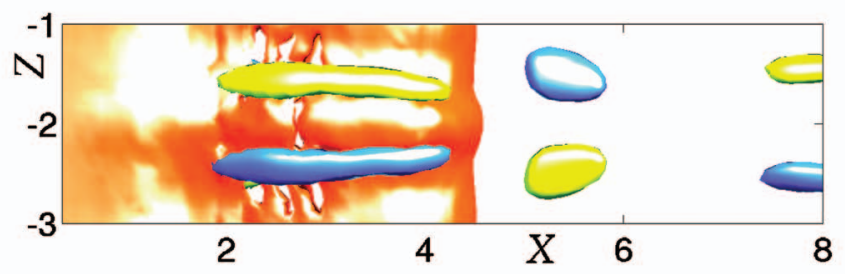

FIG. 11. (Color). Calculated isovorticity surface $\omega_{x}= \pm 0.4$ and isotempera-

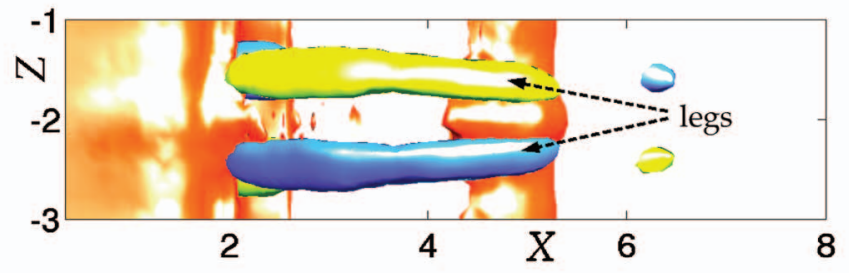
ture surface $\Theta=0.15$ at $\mathrm{Ri}=1.0$ and $\mathrm{Re}=85$. The flow is from left to right

(d) $t / T=3 / 6$ and the gravity direction is in the negative $y$ direction.

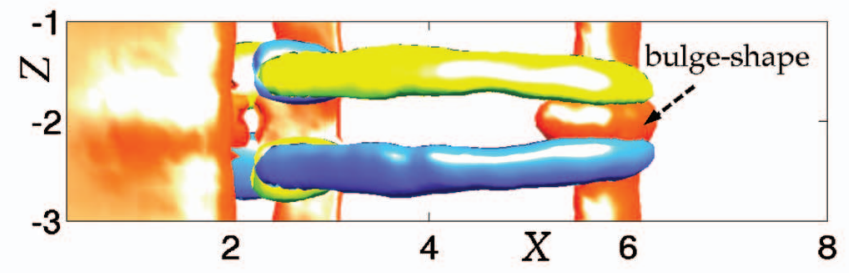

(e) $t / T=4 / 6$

(f) $t / T=5 / 6$

This suggests that the volume of the hot fluid region (bulge shape) steadily increases with time. In Fig. 14(c), a "plume shape" (P) is observed at $x=15$.

\section{B. Cross-sectional flow fields}

To gain insight into the lift-up of the bulge shape and its development towards a plume shape, the evolution of the upper vortex core is followed in time and space. The calculated temperature and velocity fields are presented at different streamwise cross sections in Fig. 15.

For $t=0$, the core region of the upper vortex is at $x$ $=7.5$. The cross-sectional temperature field $\Theta(y, z)$ is shown in Fig. 15(a). It is observed that most of the hot fluid resides inside the vortex core around $y=0.5$. Not surprisingly, the same bulge shape is found at the in-plume position $(z=-2)$. Furthermore, a nonuniform temperature distribution is observed in the upper vortex along the spanwise direction.

Correspondingly, in Fig. 15(e), the velocity cross section shows pairs of counter-rotating vortices (CRV) around the bulge region $(y=1.5)$ above the upper vortex. The position of the CRV coincides with the legs of the $\Lambda$-shaped structure, which can be seen in Fig. 13(a). Between the regions of the counter-rotating vortices, hot fluid is lifted up, which is revealed from the temperature field in Fig. 14(a). This lift-up phenomenon confirms the hypothesis sketched in Fig. 16.

For $t=T / 4$, the core region of the upper vortex is at $x$ 


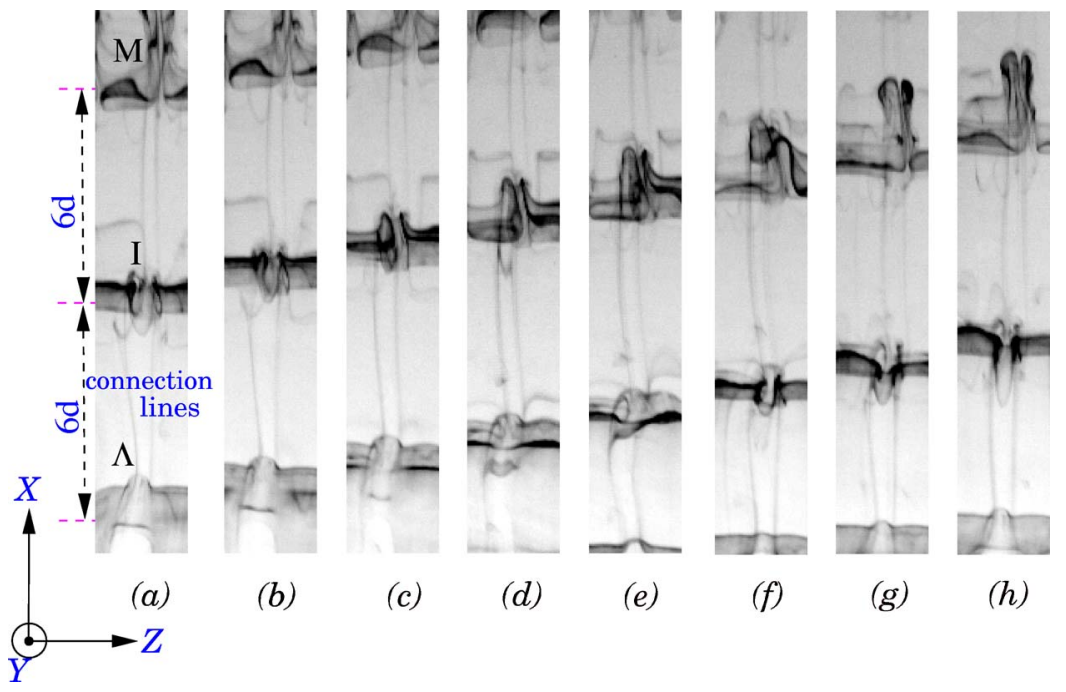

FIG. 12. (Color online). Oblique top view of the wake flow pattern for successive time steps of $\Delta t=T / 8$, obtained by tin-precipitation visualization at $\mathrm{Re}=117$ and $\mathrm{Ri}=1.0$. The flow is from bottom to top and the gravity direction is in the negative $y$ direction. $\Lambda$ : the $\Lambda$-shaped structure; $\mathbf{I}$ : the intermediate stage; and $\mathbf{M}$ : the mushroom-type structure.
=9.0. From the temperature field [Fig. 15(b)], it is observed that the hot fluid region is elongated in vertical direction. In side view, it is noticed that the bulge shape is inclined under a certain angle. From the observation, the lifted-up fluid is still connected with the vortex core.

Furthermore, the corresponding cross-sectional velocity field shows a strong upward motion in Fig. 15(f) at $y=2.0$ at the in-plume position. This upward motion is caused by the buoyancy force. The height of the stem increases with time $(y=1.5)$ [see Fig. 15(f)].

Besides the upward motion at $z=-2$, downward motions are observed at the out-of-plume position $(z=-1,-3)$. It can be seen that these upward and downward motions create layers of shear flow. The regions of shear flow coincide with the CRV. On the other hand, the CRV enhance the elongation of the bulge shape and the route towards the plume shape. For $t=2 T / 4$, the core region of the upper vortex is at $x=10.5$. The lifted-up fluid continues to move upwards and the CRV are also stretched $(y=2.0)$, as shown in Fig. 15(g).

When the upper vortex is convected at $x=12.0$, it seems that [see Fig. 15(d)] the vortex core experiences a spanwise deformation. Furthermore, it is observed that a second pair of counter-rotating vortices appears at $y=1.5$ just above the upper vortex, as shown in Fig. 15(h), and the primary CRV continues to move upwards, at $y \simeq 2.5$. It is believed that the increased height of the primary CRV coincides with the cap of the mushroom-type structure. ${ }^{14}$

\section{SUMMARY AND CONCLUSIONS}

The 3D flow transition behind a heated cylinder is analyzed at low Reynolds numbers: $\operatorname{Re}=\mathcal{O}(100)$. Both visualizations and numerical simulations show that the transition manifests itself in the form of mushroom-type structures in the far wake and of $\Lambda$-shaped structures in the near wake. The spanwise distance between the $\Lambda$-shaped structures and, as a logical consequence, between the escaping mushroomtype structures is about two cylinder diameters.

Additionally, it has been observed that the $\Lambda$-shaped structures consist of two legs and a head. This also holds for the transition to turbulence in boundary layers, where the $\Lambda$-shaped structures are often referred to as "hairpin" or "horseshoe" vortices. Klebanoff et al. ${ }^{23}$ artificially generated TS waves by an electromagnetically vibrating ribbon in their
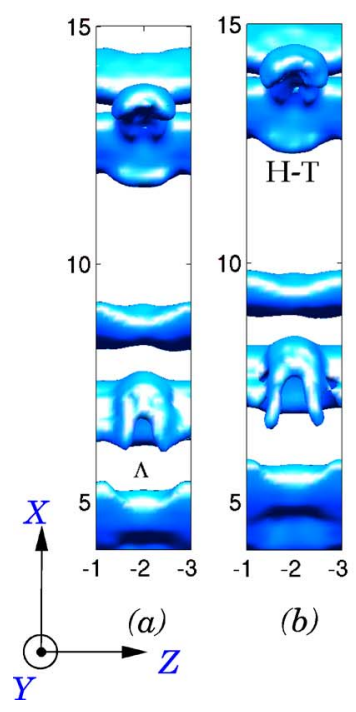

(b)

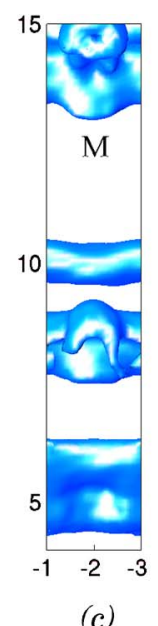

(c)

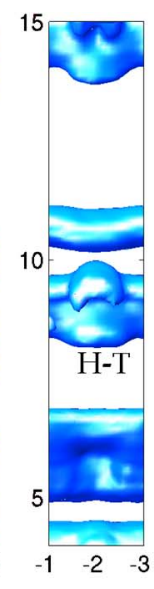

(d)

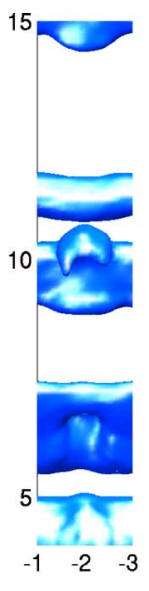

(e)

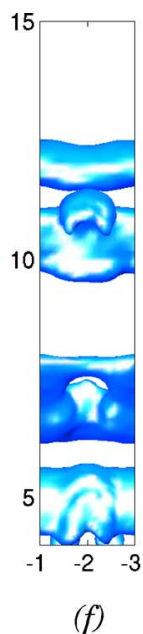

(f)

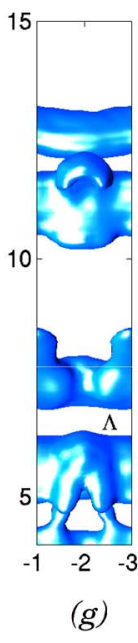

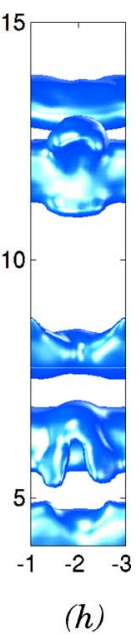

FIG. 13. (Color online). Calculated iso- $\lambda_{2}$ surface $\lambda_{2}=-0.05$ as a function of time, $\Delta t=T / 8$, at $\mathrm{Re}=85$ and $\mathrm{Ri}$ $=1.0$. The flow is from bottom to top and the gravity direction is in the negative $y$ direction. H-T: the halftorus shape structure; $\mathbf{M}$ : the mushroom-type structure. 

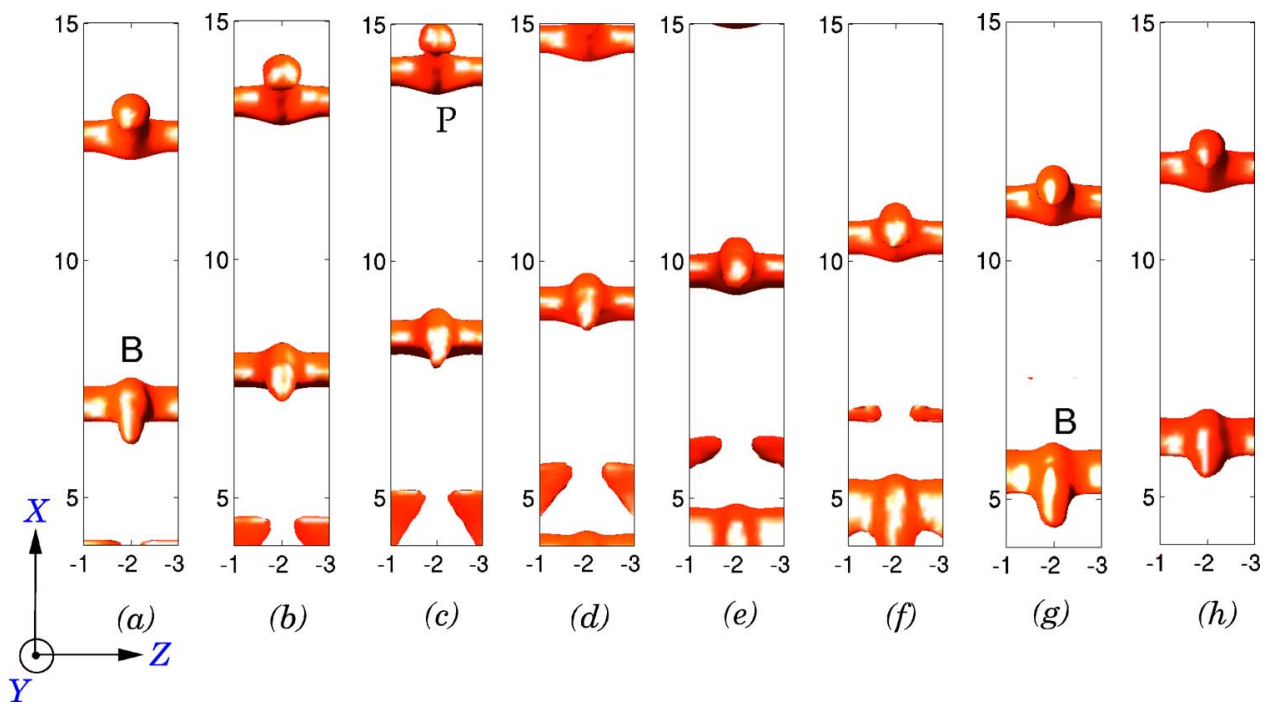

FIG. 14. (Color online). Top view of numerically calculated isotemperature surface $\Theta=0.1$ for $\mathrm{Ri}=1.0$ and $\mathrm{Re}$ $=85$. The flow is from bottom to top and gravity direction is in the negative $y$ direction. B: the bulge shape; $\mathbf{P}$ : the plume shape.

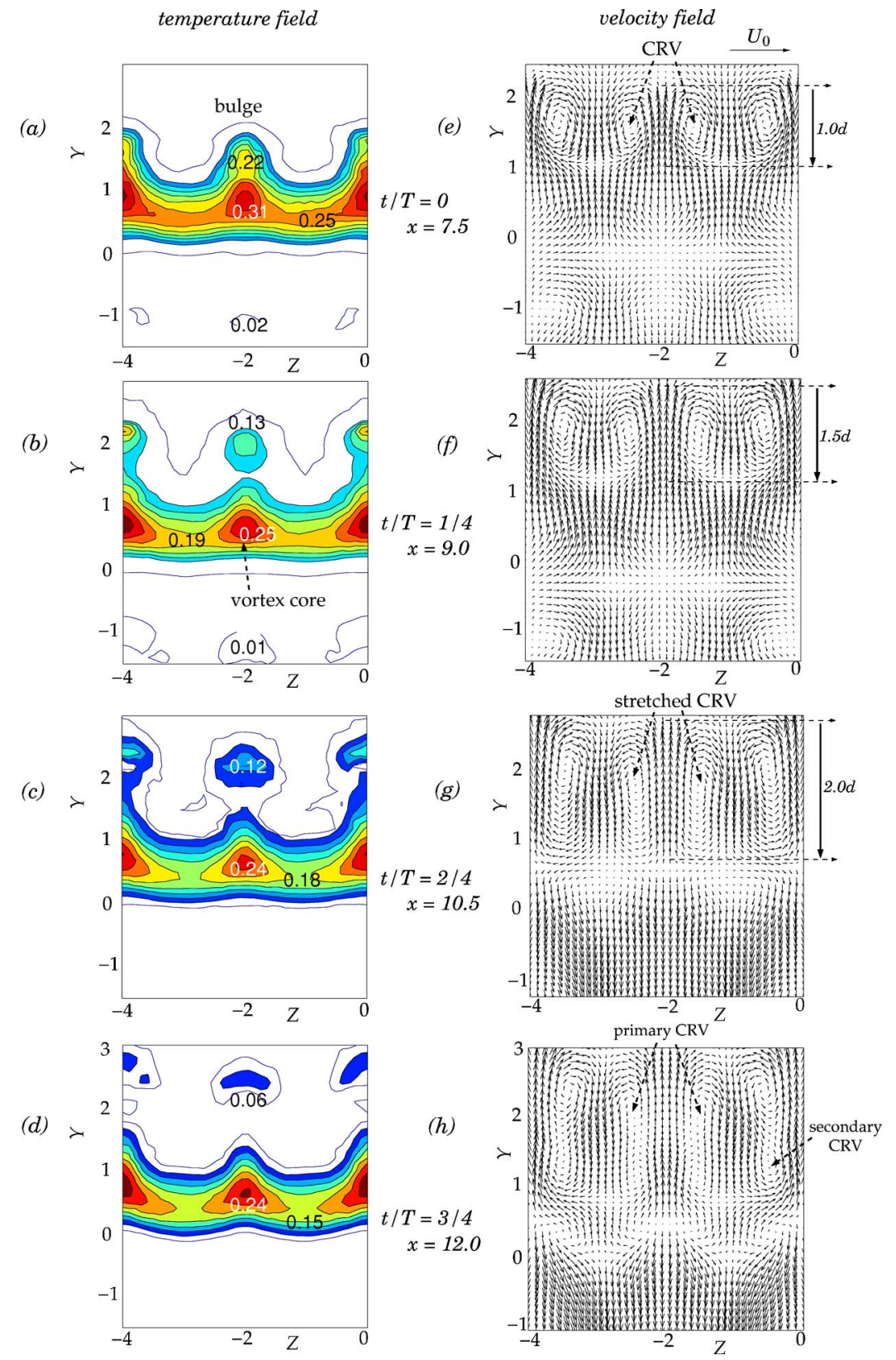

FIG. 15. (Color online). The development of the upper vortex for $\mathrm{Ri}=1.0$ and $\mathrm{Re}=85$. Left: temperature field; right: velocity field. CRV: counter-rotating vortices. 


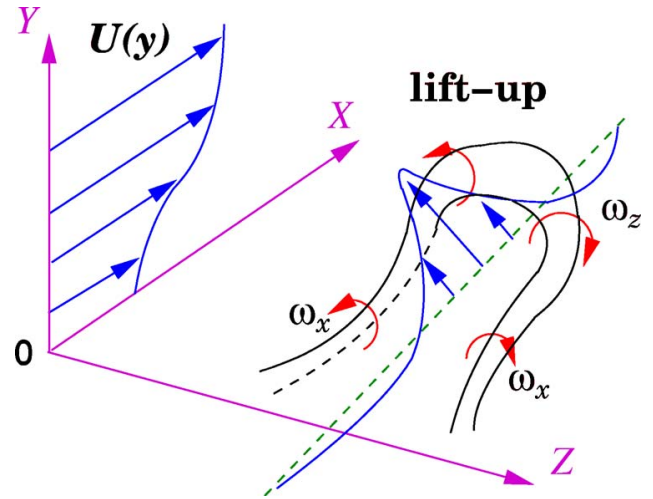

FIG. 16. (Color online). Sketch of the lift-up process of the $\Lambda$-shaped structure.

experiments. The 3D disturbances grew rapidly and resulted in an array of $\Lambda$-shaped structures (Bayly et al. ${ }^{24}$ ). Furthermore, $\Lambda$-shaped structures are also observed in a mixing layer flow by Pierrehumbert and Widnall ${ }^{25}$ and Corcos and Lin. ${ }^{26}$ It suggests that the occurrence of $\Lambda$-shaped structures is a ubiquitous effect and seems to be the crucial step in the transition from laminar to turbulent flow.

Although the legs of the $\Lambda$-shaped structure seem to coincide with streamwise vorticity regions, the origin of the head is critical in understanding the formation of the $\Lambda$-shaped structure. It is rather difficult to say when the $\Lambda$-shaped structure is exactly formed. However, the bulge shape in the temperature field suggests that the fluid is being moved upwards between the legs of the $\Lambda$-shaped structure, as sketched in Fig. 16. However, when exactly the $\Lambda$-shaped structure forms, is still not clear. It is possible that the $\Lambda$-shaped structure is already generated close behind the cylinder. However, some investigators suggest a "bridge process" of the streamwise vorticity regions to happen in a highstrain region, which is investigated in the context of boundary layer transition (Zhou et al. ${ }^{27}$ ).

In general, it seems that the $\Lambda$-shaped structure is accompanied with the occurrence of low-speed streaks. Lowmomentum fluid is accumulated between the two legs. The lift-up of low-momentum fluid between the streamwise legs is essential in the formation of the $\Lambda$-shaped structure, as sketched in Fig. 16. The lift-up process takes place at the regions between the legs and the head. As a result, hot fluid is being pulled out of the upper vortex core. In the meantime, the hot fluid will be stretched upwards due to the buoyancy force.

It seems that the escape of mushroom-type structures from the upper vortices is a combined effect. In the early stage of the evolution, streamwise vorticity plays an important role, while buoyance becomes more and more important after hot fluid is being pulled out of the vortex core between the legs of the $\Lambda$-shaped structures. The generation of streamwise vorticity in the near wake is mainly attributed to baroclinic vorticity production in the near wake, which is extensively studied in another paper [M. Ren, C. C. M. Rindt, and A. A. van Steenhoven, "Cyclic-process in a heated-cylinder wake flow," J. Fluid Mech. (submitted)] as a cyclic process. It appears that streamwise vorticity induces the occurrence of wall-normal vorticity, which consequently leads to spanwise temperature differences. This spanwise temperature gradient leads to streamwise vorticity by the action of baroclinic vorticity production. This cyclic process might be related to the mode-A instability, which is understood as a self-sustaining process (previously formed streamwise vorticity affects the formation of new streamwise structures).

In summing up, the transition mechanism is characterized by two coherent structures $(\Lambda$ and $\mathbf{M})$. An intermediate stage is observed between the evolution of $\Lambda$-shaped structures and the escaping mushroom-type structures. Furthermore, this intermediate step is characterized as a lift-up process (Fig. 8), which takes place spatially between the legs and head of the $\Lambda$-shaped structure. Due to this lift-up, the mushroom-type structure is generated in the form of a "cap," which is a vortex ring forming at the advancing front.

\section{ACKNOWLEDGMENTS}

This work is part of the research programs of the Netherlands Foundation for Fundamental Research on Matters (FOM), which is financially supported by the Netherlands Organization for Scientific Research (NWO). The authors appreciate the support of the technical staff of the Laboratory for Energy Technology of the Department of Mechanical Engineering at Eindhoven University of Technology.

${ }^{1}$ C. H. K. Williamson, "Three-dimensional wake transition,” J. Fluid Mech. 328, 345 (1996).

${ }^{2}$ R. Mittal and S. Balachander, "Generation of streamwise vortical structures in bluff-body wake," Phys. Rev. Lett. 75, 1300 (1995).

${ }^{3}$ C. H. K. Williamson, "The existence of two stages in the transition to three-dimensionality of a cylinder wake," Phys. Fluids 31, 3165 (1988).

${ }^{4}$ H. Zhang, U. Fey, B. R. Noack, M. König, and H. Eckelmann, "On the transition of cylinder wake," Phys. Fluids 7, 779 (1995).

${ }^{5}$ J. C. Lecordier, L. Hamma, and P. Paranthoën, "The control of vortex shedding behind heated circular cylinders at low Reynolds number," Exp. Fluids 10, 224 (1991).

${ }^{6}$ A. Wang, Z. Travnicek, and K. Chia, "On the relationship of effective Reynolds number and Strouhal number for the laminar vortex shedding of a heated circular cylinder," Phys. Fluids 12, 1401 (2000).

${ }^{7}$ J. C. Lecordier, L. Browne, S. Le Masson, F. Dumouchel, and P. Paranthoën, "Control of vortex shedding by thermal effect at low Reynolds numbers," Exp. Therm. Fluid Sci. 21, 227 (2000).

${ }^{8}$ R. N. Kieft, C. C. M. Rindt, and A. A. van Steenhoven, "Heat induced transition of a stable vortex street," Int. J. Heat Mass Transfer 45, 2739 (2002).

${ }^{9}$ A. A. van Steenhoven and C. C. M. Rindt, "Flow transition behind a heated cylinder," Int. J. Heat Fluid Flow 24, 322 (2003).

${ }^{10}$ R. N. Kieft, C. C. M. Rindt, A. A. van Steenhoven, and G. J. F. van Heijst, "On the wake structure behind a heated horizontal cylinder in cross-flow," J. Fluid Mech. 486, 189 (2003).

${ }^{11}$ W. J. P. M. Maas, C. C. M. Rindt, and A. A. van Steenhoven, "The influence of heat on the 3D-transition of the von Kármán-vortex street," Int. J. Heat Mass Transfer 46, 3069 (2003)

${ }^{12}$ M. Ren, C. C. M. Rindt, and A. A. van Steenhoven, "Experimental and numerical investigation of the vortex formation process behind a heated cylinder," Phys. Fluids 16, 3103 (2004).

${ }^{13}$ R. N. Kieft, "Mixed convection behind a heated cylinder," Ph.D. thesis, Eindhoven University of Technology, Eindhoven, The Netherlands, 2000.

${ }^{14}$ M. Ren, "3D flow transition behind a heated cylinder," Ph.D. thesis, Eindhoven University of Technology, Eindhoven, The Netherlands, 2005.

${ }^{15}$ M. Ren, C. C. M. Rindt, and A. A. van Steenhoven, "3D vortices in the wake flow behind a heated cylinder," Int. J. Transp. Phenom. 6, 177 (2004). 
${ }^{16} \mathrm{H}$. Honji, S. Taneda, and M. Tatasuno, "Some practical details of the electrolytic precipitation method of flow visualization," Rep. Res. Inst. Appl. Mech. (Kyushu Univ.) 28, 83 (1980).

${ }^{17}$ Y. Maday, A. Patrta, and E. Ronquist, "An operator-integration-factor splitting method for time-dependent problems: Applications to compressible flow," J. Sci. Comput. 5, 263 (1990).

${ }^{18}$ L. J. P. Timmermans, P. D. Minev, and F. N. van de Vosse, "An approximate projection scheme for incompressible flow using spectral elements," Int. J. Numer. Methods Fluids 22, 673 (1996).

${ }^{19}$ G. E. Karniadakis and G. S. Triantafyllou, "Three-dimensional dynamics and transition to turbulence in the wake of bluff bodies," J. Fluid Mech. 238, 1 (1992).

${ }^{20}$ F. N. van de Vosse, P. D. Minev, and L. J. P. Timmermans, "A spectral element projection scheme for incompressible flow with application to shear-layer stability studies," Houst. J. Math 34, 1284 (1995).

${ }^{21}$ J. Joeng and F. Hussain, "On the identification of a vortex," J. Fluid Mech. 285, 69 (1995).
${ }^{22}$ M. S. Acalar and C. R. Smith, "A study of hairpin vortices in a laminar boundary layer. Part 1. Hairpin vortices generated by hemisphere protuberance," J. Fluid Mech. 175, 1 (1987).

${ }^{23}$ P. S. Klebanoff, K. D. Tidstrom, and L. M. Sargent, "The threedimensional nature of boundary-layer instability," J. Fluid Mech. 12, 1 (1962).

${ }^{24}$ B. J. Bayly, S. A. Orszag, and T. Herbert, "Instability mechanisms in shear-flow transition," J. Fluid Mech. 184, 207 (1987).

${ }^{25}$ R. T. Pierrehumbert and S. E. Widnall, "The two- and three-dimensional instabilities of a spatially periodic shear layer," J. Fluid Mech. 114, 59 (1982).

${ }^{26}$ G. M. Corcos and S. J. Lin, "The mixing layer: deterministic models of a turbulent flow. Part 2. The origin of the three-dimensional motion," J. Fluid Mech. 139, 67 (1984).

${ }^{27}$ J. Zhou, R. J. Adrian, and S. Balachandar, "Autogeneration of near wall vortical structure in channel flow," Phys. Fluids 8, 288 (1996). 